\title{
Factors Affecting the Cost of Fissure Sealants: A Dental Insurer's Perspective
}

\author{
Stephen A. Eklund, DDS, DrPH \\ Program in Dental Public Health \\ School of Public Health \\ The University of Michigan \\ Ann Arbor, MI 48109-2029
}

\begin{abstract}
The slow adoption of sealants into dental practice is attributed partly to the fact that few prepayment plans pay for sealants. An important concern of insurers is how a sealants benefit will affect premiums.

In this analysis, caries rates for various surfaces and teeth, failure rates for both sealants and amalgam restorations, fees and copayments, interest rates, reimbursement policies for replacement and repair, and the passage of time were studied to assess the cost to an insurer of amalgam restorations versus sealants.

The results indicate:

1. Sealants as a covered benefit for molars may not cause premiums to increase if fees and copayment levels are properly balanced. If caries in children continues to decline, however, higher premiums may be required if a sealant benefit is provided.

2. Sealants as a covered benefit for bicuspids are likely to require higher premiums.

3. The level of dental caries in the population should be monitored closely, because a further substantial decline in caries prevalence could diminish the economic argument for sealants.
\end{abstract}

Key Words: sealants, dental caries, prepayment

\section{Introduction}

There is ample evidence that dental sealants, when properly applied and maintained, effectively prevent dental caries in pits and fissures (1). Despite this evidence, the widespread adoption of sealants has been slow to come (2). One reason is that sealants are rarely a covered benefit in dental prepayment plans (3). An important concern of the insurer is how sealants, if a covered benefit, will affect the premium. The primary purpose of this paper is to look at sealants as a covered benefit from the point of view of an insurance company to determine whether premiums are likely to change because of the addition of such a benefit.

\section{Methods-Variables}

The approach used was to follow the expected caries occurrence of a cohort of children through a period of eight years, and to calculate the dollar cost to an insurance company of the required treatment, both with and without sealants. Specific variables used are:

1. Dental caries. The tooth- and surface-specific caries and restoration data are from the First National Health and Nutrition Examination Survey (NHANES I) (4) and the Southwestern component of the Hispanic Health and Nutrition Examination Survey (HHANES) (5). A detailed account appeared in the spring issue of this journal (6). The forms of the data are 1) the change in the percent of occlusal "successes" each year-that is, the number of occlusal lesions per 100 teeth that become carious each year but where there is no proximal lesion on the tooth; 2) the percent of teeth that develop buccal or lingual lesions each year; and 3) the percent of teeth that develop at least one proximal lesion each year (the "failures"). These "failures" are not to be confused with the failures on the percent-effectiveness equation as described by Baskin and Ryge (7). In that context a failure can occur only in a clinical trial with intrasubject control teeth, where the sealed surface is carious, and the contralateral control surface is sound.

The values appearing in Table 1 are the averages of these values for the eight (first and second) molar teeth, and the averages for the eight bicuspid teeth. While there are some intertooth differences $(6)$, each tooth type (molars and bicuspids) is enough alike that it is reasonable to group them together. For all of these variables, the "year 0 " is the first age at which more than 50 percent of the teeth of that type have erupted. For first molars, that age is six years; for second molars, 12 years; for first bicuspids, 10 years; and for second bicuspids, 11 years (8). These values provide the expected yearly increment following eruption of the various types of carious lesions and, therefore, form the basis for assessing the dollar cost through time of the alternatives of amalgam restoration and sealants. These data are as derived from NHANES I and HHANES (6) and show some minor fluctuations from year to year. If "smoothed" data are used instead, the results of the analysis are not changed in any material way.

NHANES I data are used because they are the most recent national data that provide surface data and include a wide enough age range for this analysis. HHANES data have also been used as representative of a lower prevalence population. Although not a statistical sample of the United States population, it has been shown that the children in the Southwestern component of the HHANES exhibit dental caries levels that are remarkably similar to all children from that region at approximately the same time (9). Data from the Children's Survey (10), while covering only children up to age 17, would also be interesting to compare to these NHANES I data. Detailed surface-specific data from that study, however, have not yet been published or released for public use. 
TABLE 1

Selected Caries Increments for Molars and Bicuspids: United States, 1971-74 (NHANES I) and the Southwestern US Hispanic Population, 1982-84 (HHANES)

\begin{tabular}{|c|c|c|c|c|c|c|c|c|c|}
\hline & \multicolumn{9}{|c|}{ Posteruption Years } \\
\hline & 0 & 1 & 2 & 3 & 4 & 5 & 6 & 7 & 8 \\
\hline \multicolumn{10}{|c|}{ Molars (NHANES I) } \\
\hline Successes ${ }^{*}$ & 8.4 & 9.1 & 13.7 & 4.2 & 10.7 & 0.0 & 3.3 & 0.5 & 1.8 \\
\hline B or Lt & 1.4 & 1.6 & 1.6 & 4.2 & 1.2 & 1.4 & 0.4 & 1.2 & 1.2 \\
\hline Failuresf & 0.2 & 0.4 & 0.8 & 1.8 & 1.9 & 3.2 & 1.1 & 2.0 & 1.4 \\
\hline \multicolumn{10}{|c|}{ Bicuspids (NHANES I) } \\
\hline Successes & 1.6 & 1.3 & 2.1 & 1.6 & 0.0 & 1.0 & 0.8 & 0.0 & 0.0 \\
\hline Failures & 0.8 & 0.1 & 1.1 & 0.8 & 0.6 & 3.2 & 1.7 & 3.1 & 0.3 \\
\hline \multicolumn{10}{|c|}{ Molars (HHANES) } \\
\hline Successes & 4.9 & 7.2 & 3.2 & 10.5 & 2.3 & 6.0 & 2.3 & 2.4 & 0.0 \\
\hline $\mathrm{B}$ or $\mathrm{L}$ & 2.4 & 3.2 & 3.3 & 2.8 & 2.3 & 4.6 & 1.4 & 3.7 & 0.9 \\
\hline Failures & 0.1 & 0.2 & 0.0 & 0.4 & 0.9 & 1.9 & 0.2 & 1.7 & 0.0 \\
\hline \multicolumn{10}{|c|}{ Bicuspids (HHANES) } \\
\hline Successes & 0.8 & 0.4 & 0.4 & 1.1 & 0.8 & 0.6 & 1.3 & 0.1 & 0.2 \\
\hline Failures & 0.6 & 0.0 & 0.6 & 0.1 & 0.5 & 0.7 & 0.8 & 0.3 & 1.1 \\
\hline
\end{tabular}

*Incremental number of teeth per 100 teeth with occlusal decay or filling, without proximal involvement. Value for each year is the increment above the level of the previous year.

tincremental number of teeth per 100 teeth with buccal or lingual decay or filling.

fincremental number of teeth per 100 teeth with at least one proximal decayed or filled surface.

Source: National Center for Health Statistics: Special dental data tape from the Health and Nutrition Examination Survey, United States, 1971-74, and Southwestern component of the Hispanic Health and Nutrition Examination Survey, $1982-84$.

2. Fees. Estimates of fees for fillings and sealants are used with the caries data to determine the effect of a sealant benefit on the overall cost of treatment. For the sealant alternative, the cost of the initial sealant is attributed to the first vear after eruption. For the alternative without sealants, the cost of the amalgam restoration is attributed to the year in which it would be required, based on the data in Table 1 . Lesions in year zero are assumed to have occurred before the first posteruptive clinical visit, and thus to require a restoration under both alternatives. The costs of such restorations (as well as all proximal restorations) are equivalent between the alternatives and are excluded from the cost analysis. The fee value that is used in the analysis is the ratio of the one-surface amalgam fee to the single-tooth sealant fee. The starting fee ratio of $2: 1$ is based on the 1982 Fee Survey of the American Dental Association (11), where the mean fee for a single-surface amalgam is $\$ 21.21$ and the mean fee for a sealant is $\$ 11.14$. Values for these and the remaining variables are displayed in Tables 2 and 3.

3. Copayment lecels. Closely related to fees is the level of copayment for the services. The copayment levels of 10 and 50 percent represent the usual range for preventive and restorative procedures.

4. Replacement and repair policy. Of additional relevance to fees and copayment is the policy on replacement or repair. There are a variety of possibilities for reimbursement of failed restorations and sealants that can affect the cost $t$ o the carrier. The four possibilities con- sidered are: 1) the carrier does not pay for replacement or repair of restorations or sealants; 2 ) the carrier does not pay for repair of sealants, but does pay for replacement of amalgams and for restoration required when a sealant fails; 3) the carrier pays for the replacement of amalgams, but bears no further financial liability for a surface that has been sealed; and 4) the insurer pays for replacement restoration and resealing whenever necessary.

5. Failure rates. Also of importance are the anticipated failure rates for sealants and amalgam restorations. For restorations, what little data are available have recently been reviewed by Maryniuk (12). While data specific to single surface restorations are sparse, the failure rates in the first few years after placement appear to be low, possibly no more than one or two percent per year. Nevertheless, because of the uncertainty surrounding amalgam failure rates, the potential effects of rates in the range from 0.5 percent to 5.0 percent/per year are assessed. For sealants, too, the data are difficult to pin down because many of the studies in the literature used materials that are not as successful as newer materials. The values in this analysis, from five to 10 percent in the first year and from two to five percent after the first year, are based on the recent review of the topic by Mertz-Fairhurst (1), and reflect data from the use of newer materials.

6. Interest (discount) rates. With the need for restorations coming at various times in the future, some interest, or discount, rate is required. Because sealants are 
TABLE 2

Savings Ratios* for Various Alternatives of Sealants vs. Amalgams: Molars

\begin{tabular}{|c|c|c|c|c|c|c|c|c|c|c|c|c|}
\hline \multirow[b]{2}{*}{ Variable } & \multicolumn{9}{|c|}{ NHANES I (1971-74) } & \multicolumn{3}{|c|}{ HHANES (1982-84) } \\
\hline & A & B & C & $\mathrm{D}$ & $\mathrm{E}$ & F & G & $\mathrm{H}$ & I & $\mathrm{J}$ & $\mathrm{K}$ & $\mathrm{L}$ \\
\hline $\begin{array}{l}\text { Ratio of filling fee } \\
\text { to sealant fee (ini- } \\
\text { tial) }\end{array}$ & $2: 1$ & $2: 1$ & $4: 3$ & $1: 1$ & $2: 1$ & $2: 1$ & $2: 1$ & $2: 1$ & $2: 1$ & $2: 1$ & $1: 1$ & $2: 1$ \\
\hline $\begin{array}{l}\text { Ratio of filling fee } \\
\text { to sealant fee (re- } \\
\text { placement) }\end{array}$ & $2: 1$ & $2: 1$ & $4: 3$ & 1:1 & $2: 1$ & $4: 1$ & $2: 1$ & $2: 1$ & $2: 1$ & $2: 1$ & $1: 1$ & $2: 1$ \\
\hline Sealant copay & $10 \%$ & $10 \%$ & $10 \%$ & $10 \%$ & $50 \%$ & $10 \%$ & $10 \%$ & $10 \%$ & $10 \%$ & $10 \%$ & $10 \%$ & $50 \%$ \\
\hline Filling copay & $10 \%$ & $10 \%$ & $10 \%$ & $10 \%$ & $10 \%$ & $10 \%$ & $10 \%$ & $10 \%$ & $10 \%$ & $10 \%$ & $10 \%$ & $10 \%$ \\
\hline $\begin{array}{l}\text { Sealant failure rate } \\
\text { (year 1) }\end{array}$ & $10 \%$ & $10 \%$ & $10 \%$ & $10 \%$ & $10 \%$ & $10 \%$ & $5 \%$ & $5 \%$ & $10 \%$ & $10 \%$ & $10 \%$ & $10 \%$ \\
\hline $\begin{array}{l}\text { Sealant failure rate } \\
\text { (>year } 1)\end{array}$ & $5 \%$ & $5 \%$ & $5 \%$ & $5 \%$ & $5 \%$ & $5 \%$ & $5 \%$ & $2 \%$ & $5 \%$ & $5 \%$ & $5 \%$ & $5 \%$ \\
\hline $\begin{array}{l}\text { Occlusal amalgam } \\
\text { failure rate (per } \\
\text { year) }\end{array}$ & $2 \%$ & $2 \%$ & $2 \%$ & $2 \%$ & $2 \%$ & $2 \%$ & $2 \%$ & $2 \%$ & $1 \%$ & $2 \%$ & $2 \%$ & $2 \%$ \\
\hline $\begin{array}{l}B \text { or } L \text { amalgam } \\
\text { failure rate (per } \\
\text { year) }\end{array}$ & $2 \%$ & $2 \%$ & $2 \%$ & $2 \%$ & $2 \%$ & $2 \%$ & $2 \%$ & $2 \%$ & $.5 \%$ & $2 \%$ & $2 \%$ & $2 \%$ \\
\hline Discount rate & $10 \%$ & $5 \%$ & $10 \%$ & $10 \%$ & $10 \%$ & $10 \%$ & $10 \%$ & $10 \%$ & $10 \%$ & $10 \%$ & $10 \%$ & $10 \%$ \\
\hline $\begin{array}{l}\text { Results } \\
\text { Savings } \\
\text { ratio } 1^{*}\end{array}$ & .93 & 1.07 & .62 & .47 & 1.68 & .93 & .93 & .93 & .93 & .87 & .43 & 1.56 \\
\hline $\begin{array}{l}\text { Savings } \\
\text { ratio } 2 \dagger\end{array}$ & .76 & .83 & .55 & .43 & 1.15 & .76 & .78 & .87 & .74 & .71 & .40 & 1.09 \\
\hline $\begin{array}{l}\text { Savings } \\
\text { ratio } 3^{* *}\end{array}$ & 1.00 & 1.16 & .67 & .50 & 1.81 & 1.00 & 1.00 & 1.00 & .97 & .93 & .46 & 1.67 \\
\hline $\begin{array}{l}\text { Savings } \\
\text { ratio } 4 \ddagger\end{array}$ & .76 & .84 & .51 & .38 & 1.37 & .87 & .80 & .88 & .73 & .70 & .35 & 1.26 \\
\hline
\end{tabular}

* Savings ratio 1 is the ratio of the dollars that would have been spent on the occlusal and buccal surfaces divided by the cost of sealing those same surfaces, assuming the carrier does not pay for failures or replacements of either the fillings or sealants.

+ Savings ratio 2 is the same ratio, assuming failures of both sealants and fillings, and all failures are filled with amalgam when carious, and these are paid for by the carrier.

* Savings ratio 3 is the same ratio, assuming that the carrier pays for replacing failed amalgams, but bears no liability for the sealed surface. $\ddagger$ Savings ratio 4 is the same ratio, assuming that the insurer pays for fillings and resealing whenever necessary.

most frequently placed before the sealed surface becomes clinically carious, the time between the placement of the sealant and the time that surface would become clinically carious (without a sealant) is of economic importance. That time represents money, and must be viewed relative to the anticipated cost of money. The values of five and 10 percent used in this analysis represent a reasonable range in the present economic climate. Net present value of the cost of each alternative is calculated based on the timing of the costs and the interest rate.

With each of these variables, only a few values, representing a realistic range, are presented in Tables 2 and 3 . These values are nevertheless sufficient to demonstrate the effect of changes in these variables on the cost of sealants to a carrier. 
TABLE 3

Savings Ratios* for Various Alternatives of Sealants vs. Amalgams: Bicuspids

\begin{tabular}{|c|c|c|c|c|c|c|c|c|c|c|c|}
\hline \multirow[b]{2}{*}{ Variable } & \multicolumn{8}{|c|}{ NHANES I (1971-74) } & \multicolumn{3}{|c|}{ HHANES (1982-84) } \\
\hline & A & B & C & $\mathrm{D}$ & E & F & G & $\mathrm{H}$ & I & $\mathrm{J}$ & K \\
\hline $\begin{array}{l}\text { Ratio of filling fee to sealant } \\
\text { fee (initial) }\end{array}$ & $2: 1$ & $2: 1$ & $4: 3$ & $2: 1$ & $2: 1$ & $2: 1$ & $2: 1$ & $2: 1$ & $2: 1$ & $4: 3$ & $2: 1$ \\
\hline $\begin{array}{l}\text { Ratio of filling fee to sealant } \\
\text { fee (replacement) }\end{array}$ & $2: 1$ & $2: 1$ & $4: 3$ & $2: 1$ & $4: 1$ & $2: 1$ & $2: 1$ & $4: 1$ & $2: 1$ & $4: 3$ & $4: 1$ \\
\hline Sealant copay & $10 \%$ & $10 \%$ & $10 \%$ & $50 \%$ & $10 \%$ & $10 \%$ & $10 \%$ & $50 \%$ & $10 \%$ & $10 \%$ & $50 \%$ \\
\hline Filling copay & $10 \%$ & $10 \%$ & $10 \%$ & $10 \%$ & $10 \%$ & $10 \%$ & $10 \%$ & $10 \%$ & $10 \%$ & $10 \%$ & $10 \%$ \\
\hline Sealant failure rate (year 1 ) & $10 \%$ & $10 \%$ & $10 \%$ & $10 \%$ & $10 \%$ & $5 \%$ & $10 \%$ & $5 \%$ & $10 \%$ & $10 \%$ & $5 \%$ \\
\hline Sealant failure rate (>year 1 ) & $5 \%$ & $5 \%$ & $5 \%$ & $5 \%$ & $5 \%$ & $5 \%$ & $5 \%$ & $5 \%$ & $5 \%$ & $5 \%$ & $5 \%$ \\
\hline $\begin{array}{l}\text { Occlusal amalgam failure } \\
\text { rate (per year) }\end{array}$ & $2 \%$ & $2 \%$ & $2 \%$ & $2 \%$ & $2 \%$ & $2 \%$ & $1 \%$ & $5 \%$ & $2 \%$ & $2 \%$ & $5 \%$ \\
\hline $\begin{array}{l}B \text { or } L \text { amalgam failure rate } \\
\text { (per year) }\end{array}$ & $2 \%$ & $2 \%$ & $2 \%$ & $2 \%$ & $2 \%$ & $2 \%$ & $.5 \%$ & $5 \%$ & $2 \%$ & $2 \%$ & $5 \%$ \\
\hline Discount rate & $10 \%$ & $5 \%$ & $10 \%$ & $10 \%$ & $10 \%$ & $10 \%$ & $10 \%$ & $5 \%$ & $10 \%$ & $10 \%$ & $5 \%$ \\
\hline $\begin{array}{l}\text { Results } \\
\text { Savings } \\
\text { ratio } 1^{*}\end{array}$ & .11 & .12 & .07 & .19 & .11 & .11 & .11 & .22 & .07 & .04 & .15 \\
\hline $\begin{array}{l}\text { Savings } \\
\text { ratio } 2 t\end{array}$ & .11 & .13 & .07 & .19 & .11 & .11 & .11 & .25 & .07 & .05 & .16 \\
\hline $\begin{array}{l}\text { Savings } \\
\text { ratio } 3^{* *}\end{array}$ & .11 & .13 & .08 & .21 & .11 & .11 & .11 & .27 & .07 & .05 & .17 \\
\hline $\begin{array}{l}\text { Savings } \\
\text { ratio } 4 \ddagger\end{array}$ & .09 & .10 & .06 & .16 & .11 & .09 & .08 & .26 & .05 & .04 & .15 \\
\hline
\end{tabular}

* Savings ratio 1 is the ratio of the dollars that would have been spent on the occlusal and buccal surfaces divided by the cost of sealing those same surfaces, assuming the carrier does not pay for failures or replacements of either the fillings or sealants.

tSavings ratio 2 is the same ratio, assuming failures of both sealants and fillings, and all failures are filled with amalgam when carious, and these are paid for by the carrier.

** Savings ratio 3 is the same ratio, assuming that the carrier pays for replacing failed amalgams, but bears no liability for the sealed surface. $\ddagger$ Savings ratio 4 is the same ratio, assuming that the insurer pays for fillings and resealing whenever necessary.

7. Variables not included. Utilization rates, both in terms of the number of eligible children visiting a dentist and the number of dentists suggesting the use of sealants, will affect the cost of dental care. These factors are not included in the model, however, because they do not affect the payback to be expected per dollar spent for sealants. Also not included, because they do not affect this model, are changes in utilization that come from changes in copayment levels. The model says nothing about the total cost of care under the various alternatives presented. It is designed to assess how much cost saving will be realized per dollar spent for sealants. The purpose is simply to tell whether or not sealants will increase or decrease the total cost of dental care, and is not intended to assess how large that change is relative to the total cost of care. To estimate the total dollar value of such a change will require, at a minimum, highly detailed knowledge about the composition and characteristics of the groups involved.

\section{Methods-Calculations}

Given the variables just described, the analysis is based on repetitive calculations to derive the net present value of each alternative. For example, in the sealant alternative for the molars, the sealants are assumed to be applied at the first posteruptive visit. The data from NHANES I in Table 1 indicate that by that visit, for 
every 100 molars 8.4 will already have a carious occlusal surface and thus require a filling; 1.4 will have a buccal or lingual pit lesion; and an additional 0.2 will require a proximal restoration. Therefore, 90.0 percent of molars will remain to be sealed at "Year 0, ," and the cost to the insurer attributed to the present. It is likely that some insurers will reimburse for a sealant on an occlusal surface even though a buccal or lingual surface has already been restored. In that circumstance, 91.4 percent of molars would be sealed at "Year 0." While the example presented uses the figure of 90.0, the difference is so small that the outcome of the analysis is virtually unchanged regardless of which of these values is used.

The actual costs calculated into the future depend on the reimbursement policy, but in any case are attributed to the year in which they would be incurred. For example, when the insurer pays for resealing, the failure rate of sealants (together with the number of sealants placed minus the number of sealants lost to proximal caries) is used to calculate the number of sealants that will require resealing. This figure is multiplied by the dollar amount that the insurer will pay, and its net present value is computed. The same procedure is followed for each year, yielding the total net present value of the cost to the insurer for each alternative.

For the "restorations only" alternative, restorations are counted in the year they are expected to occur, and their cost to the insurer is calculated at net present value. At "Year 0 " the same 10.0 teeth would be restored as in the sealant alternative, and because the cost to the insurer for these restorations is the same under both alternatives, it does not enter the cost analysis. In "Year 1" under the "fillings only" alternative, 9.1 occlusal and 1.6 buccal or lingual pit restorations will be placed. The 0.4 proximal restorations are not included, because they are equivalent between the alternatives. The expected numbers of newly carious surfaces are handled the same way each year, and net present value is calculated.

Failures are also figured into the calculations according to the assumed failure rates, and the cost of the repair or replacement is attributed to the year it occurs.

A numerical spreadsheet computer program was used for these calculations. The spreadsheet as it appears for the result in Table 2, alternative A, is in Figure $i$. A complete listing of the cell formulas is available from the author. The pertinent values were modified for each of the other alternatives presented in Tables 1 and 2.

\section{Evaluation Criteria}

The criterion for evaluation of the alternatives is the ratio of the cost to the insurance company for treatment with amalgam restorations to the cost to the insurance company for treatment of the same teeth with sealants. A ratio greater than one indicates that sealants would more than pay for themselves; thus, premiums could be reduced. A ratio of less than one indicates that the sealants cost the insurance company more than the cost of the fillings that they prevent. The alternatives evalu- ated are 1) the cost of treating the teeth with amalgam restorations compared to the cost of a single application of sealants, with no payment by the insurer for failures of either sealants or fillings [Savings ratio 1]; 2) treatment with amalgam compared to a single application of sealants, with failures of both fillings and sealants restored when necessary with amalgam and paid for by the carrier [Savings ratio 2]; treatment with amalgam compared to a single application of sealants, with no payment for the sealed surface beyond its initial placement, either for resealing or filling, but with payment for failed fillings on surfaces that were never sealed [Savings ratio 3]; and 4) the same ratio assuming reimbursement for replacement and repair as needed for both sealants and fillings [Savings ratio 4].

The period of eight years was chosen for this analysis for several reasons. First is the uncertainty in the data on caries rates and failure rates for both fillings and sealants, and the sensitivity of the model to these rates. The data are not firm enough to support quantitative estimates any further into the future. A second set of reasons is that in both the NHANES I and the HHANES data, the peak activity for occlusal caries in molars has been reached by eight years, and the rate of proximal lesions is rising quickly; thus, if savings have not been realized by that time, they are not likely to be forthcoming in the form of fillings prevented. The final reason is that with discounting, especially at the rate of 10 percent, savings that would come beyond eight years are unlikely to have much value in today's dollars and, therefore, will have little effect on the analysis.

\section{Results}

Under the first set of conditions (Table 2, column A), the savings ratio over the eight-year period from the placement of sealants on permanent molars is somewhere between 76 cents and $\$ 1.00$ for every dollar spent for sealants. These figures indicate that under these conditions the savings to be anticipated from the placement of sealants on molars is reasonably close to the cost of placing them.

Alternative $B$ cuts the discount rate from 10 percent to 5.0 percent, with the result that sealants look a bit more favorable for all four savings ratios. The reason is that the savings from the restorations prevented come in the future: the lower the discount rate, the greater the net present value of the savings. Alternatives $C$ and $D$ demonstrate the effect of higher fees for sealants. In example A, where the sealant fee is one-half the single surface amalgam fee, the savings ratios look favorable for sealants. In example D, where the fees are equal, the savings ratios are far less favorable. Example $C$ is an intermediate position.

Alternative $E$ is closely related to $A, C$, and $D$, in that while the fees are like those in $A$, the cost for sealants paid by the insurer is reduced by increasing the copayment for sealants from 10 percent to 50 percent. This demonstrates that the insurer can use copayments to help cover the dollar cost of a benefit. As with fees, the ratio of the copayments for fillings and sealants is what is important, not the actual copayment levels. 
FIGURE 1

Sample* Spreadsheet as Used to Calculate Cost of Sealants and Amalgam Restorations

\section{A} 8 C

D

1 SEAL

2 Interest rate

Relative fees

Occlusal amalgan

$B$ or $L$ amalgam

Sealant

Sealant repair

0.1

Insurance gpay

Potential successes

Occlusa?

$B$ or $L$

2

0.9

0.9

Failures (Proximal lesions)

Year 0

Year 1

Year 2

Year 3

Year 4

Year 5

Year 6

Year 7 Year 8

$\begin{array}{rrrrrrrrr}8.4 & 9.1 & 13.7 & 4.2 & 10.7 & 0 & 3.3 & 0.5 & 1.8 \\ 1.4 & 1.6 & 1.6 & 4.2 & 1.2 & 1.4 & 0.4 & 1.2 & 1.2\end{array}$

14

Failure rates $\quad Y r 1>Y r 1$

Sealant 0.10 .05

$\begin{array}{lll}0 c c l \text { amalgam } & 0.02 & 0.02\end{array}$

$B$ or $L$ amalgam

0.02

0.02

0.4

$0.8 \quad 1.8$

1.9

$3.2 \quad 1.1$

$2 \quad 1.4$

Sealant failures

occlusal

$B$ or $L$

0.10

0.15

0.02

0.02

0.02

0.02

0.19

0.23

$0.02 \quad 0.02$

0.27

0.27
0.02

0.30

0.02

0.34

0.37

B or $L$

0.91

$2.40 \quad 1.76$

3.54

0.02

0.02

0.02

0.02

Intact sealants (would be carious)

Occlusal

Buccal

0.16

0.30

0.93

0.57

1.45

2.38

1.60

2.04

$\begin{array}{llll}0.71 & 0.49 & 0.77 & 0.83\end{array}$

Failed amalgam, repiacing sealant Occlusal

$8.19 \quad 19.49 \quad 21.93$

29.09

27.64

$28.55 \quad 27.46$

27.21

$\begin{array}{lll}1.44 & 2.74 \quad 6.01\end{array}$

6.64

7.33

7.24

7.67

8.04

$B$ or $L$

$0.02 \quad 0.07$

0.10

0.17

0.20

0.25

0.28

$.00 \quad 0.01$

0.03

0.04

0.05

0.06

0.08

Failed amalgam, never sealed Occlusal

$0.18 \quad 0.46$

0.54

0.75

$0.75 \quad 0.82$

0.83

$B$ or 1

0.03

0.06

0.15

0.17

0.20

0.21

0.23

Sealant repairs

$8.96 \quad 4.91 \quad 4.66$

4.64

4.57

4.67

4.63

4.66

Cost of fillings

21.4

30.6

16.8

23.8

2.8

7.4

3.4

6

Failure cost

Filling replacing sealant

Filling replacing filling

$$
2.14
$$

$5.44 \quad 5.53$

0.43

8.49

4.74

6.25

5.35

6.46

Sealant repair cost

$$
8.96
$$

4.91

4.66

4.64

4.57

4.67

4.63

4.66

Initial sealant cost

TOTAL
90.00

90.00

NPV

INS

Initial filling cost

44.41

.

$$
28
$$

$\begin{array}{rr}81.00 \\ 84.08 \quad 100.98 \\ 2.63 & 39.97\end{array}$

$28.63 \quad 39.97$

9.71

3 Savings ratios

$$
\begin{array}{rrrr}
1 & 2 & 3 & 4 \\
0.93 & 0.76 & 1.00 & 0.76
\end{array}
$$

*For Table 2, Alternative A. Full printout of cell formulas available from the author. 
Alternative $F$ shows the anticipated value of limiting the fee for the replacement of a sealant to 50 percent of the initial fee. This affects only Savings ratio 4 , because it is the only one that assumes the insurer will pay for the repair of sealants.

Alternatives $\mathrm{G}, \mathrm{H}$, and I look at the effects of varying the rates of failure for fillings and sealants. They demonstrate that sealants look better if they are less subject to failure or if fillings are more subject to failure. The anticipated failure rates for occlusal amalgams and the newer sealant materials are so low, however, that-at least in the short run-they do not play a major role in determining the outcome of the analysis.

Alternatives J, K, and L are based on HHANES data and, except for that, correspond to alternatives A, D, and $\mathrm{E}$. The savings ratio for all three of these alternatives based on the data from a lower-prevalence population are approximately 93 percent of the corresponding values for the NHANES I population. The expected return from the placement of sealants on permanent molars is remarkably similar in these two populations, despite the considerable difference in caries prevalence between them. This similarity in expected savings is attributable to the fact that some of the decline in available "successes" in the lower-prevalence population is compensated for by fewer subsequent proximal lesions that would require an occlusal extension of the restoration, and that, because of fewer missing teeth, the buccal and lingual pit lesions that can be prevented by sealants are not as readily "lost" as the tooth is extracted.

Moving to Table 3, which is based on the anticipated caries increments for bicuspids, the savings ratios are remarkably different. The magnitude of the differences between Tables 2 and 3 emphasizes the importance of the caries rate in assessing the value of sealants. Without sufficient numbers of lesions to prevent, the dollar cost of placing the sealants can be many times the amount that can be saved. Even in Alternative $\mathrm{H}$, where all of the variables are tipped in favor of sealants, the payback in eight years from sealing bicuspids is not expected to be much more than 25 cents on the dollar.

Alternatives I, J, and K, which are based on the lower prevalence population in HHANES, correspond to Alternatives $A, C$, and $H$, respectively. For the bicuspids, the savings ratios in the lower-prevalence population are approximately 65 percent of their value in the NHANES I population. An already low return for bicuspids is markedly lower in the lower-prevalence population. The decline in prevalence of occlusal lesions in the bicuspids is not mitigated as in the molars because there are rarely any buccal or lingual pits to prevent with sealants, and the bicuspids were not subject to high levels of proximal caries or extraction in the NHANES population.

\section{Discussion}

The conditions used in the model can only be considered to be estimates. Uncertainty surrounds several aspects of the data. These data are cross-sectional so that one does not know how many teeth developing occlusal caries but without proximal caries [the potential "successes" (6)] in any one year are being canceled out (are not apparent in the data) by such teeth from previous years that are lost to proximal caries. For example, if 10 percent of the teeth developed occlusal lesions in a particular year, but during the same year some teeth that had been classified as "successes" the previous year developed proximal lesions, the apparent increase in "successes" in cross-sectional data would be reduced below the actual 10 percent. The effect would be to underestimate the benefit of sealants. For the period of time covered by this analysis (eight years posteruption), however, all indications are that this effect is not of importance. First, the rise in proximal caries does not begin until at least four years after the rise in occlusal caries (6) and even then the yearly increment is small (see "Failures" Table 1). Second, the decrease in "successes" closely parallels the declining increment in occlusal caries for any particular tooth type, indicating that the diminishing number of available "successes" is in fact attributable to a decreasing occurrence of occlusal caries in older children. A further uncertainty with the data is that the rates of failure for both sealants and amalgam restorations are not known with precision. In addition, the ratio of sealant fees to single-surface amalgam fees, particularly in the future as sealants become more widely accepted, is not known. It is likely that copayment levels and policies on payment for resealing also will influence the relative level of sealant fees.

The possibility of intentionally placing sealant on a carious lesion as an alternative to restoration also has been excluded from the analysis. This omission is primarily because of insufficient data and the lack of a consensus to suggest what proportion of carious lesions should be treated this way. The effect of such a substitution procedure on the cost to the insurer would nevertheless also be highly dependent on the relative fee and copayment levels of the two procedures.

All of these uncertainties suggest that to accept the numbers produced by this model at face value is unwise. Nevertheless, the model does provide help with the major question that this project was meant to answer: How will adding a sealant benefit affect the premium for dental insurance? In the case of the molar teeth, it seems plausible that a sealant benefit can be added without an increase in premium. There are several ways that the insurer can "hedge the bet," such as choosing a lower copayment level for the sealant and paying for resealing at a rate below that for the original sealant. On the other hand, the conditions for bicuspids are far less favorable. It is difficult to imagine how sealants for bicuspids can be provided without resulting in higher premiums.

A major finding of the analysis is that the level of anticipated disease is critical to the value of sealants. With the exception of fees, the plausible levels of the other variables are such that they are secondary to the importance of disease levels. If there are not sufficient levels of disease for a procedure to prevent, it is difficult to justify the cost of the procedure. This observation 
has been made in a more general way by Burt (13), and is the same concept that has been described for fluoride mouthrinsing by Stamm et al. (14). On this basis, if the level of disease available to prevent has fallen substantially since NHANES I, the cost analysis from the insurer's point of view will be less favorable. In fact, when data from a lower prevalence population (HHANES) are used, the anticipated savings ratios do decline relative to those from HHANES 1. The decline in the savings ratios for the molars, however, is not nearly as great as might be anticipated based on the difference in occlusal caries prevalence. The lower prevalence of proximal lesions and the fact that because of fewer missing teeth more buccal and lingual pit lesions are available to prevent, combine to compensate partially for the dramatically lower occlusal caries prevalence. How much the caries decline will affect the economic value of sealants will be answerable with more precision only if a continuing source of surface-specific caries data covering a wide range of ages (at least into early adulthood) becomes available.

It must be emphasized that just because the dollar cost for placing sealants on bicuspids (and possibly on molars if disease levels continue to fall) is likely to be greater than the money that will be saved in the short run does not necessarily mean these teeth should not be sealed. The longer term benefits, both monetary and intangible, may well be sufficient to make it a desirable service. It does mean, however, that if a group of purchasers wants such a service to be covered by its dental prepayment plan, it must be willing to pay the increased premium that will be required.

The possibility of limiting the use of sealants to the most "susceptible" teeth or individuals is appealing. Sealing molars but not bicuspids is in fact such a limited procedure. Within the molars, it would be desirable if the teeth most likely to become carious could be identified, and sealants limited to them. As appealing as such a procedure sounds, it is unlikely to be possible for insurance companies to implement in the foreseeable future. First, no clear criteria for distinguishing susceptible from immune teeth have been identified, and prospective demonstrations of the sensitivity and specificity of such criteria would have to be provided before they could be required. Second, it is difficult to imagine how a dentist would be able to refuse to provide this service, should it be a covered benefit, for a patient who requests it. Were that tooth to develop a subsequent lesion, the dentist could be in a difficult position indeed. To go bevond limits to particular types of teeth (i.e., molars) and certain age ranges is unlikely to be practical given the present level of knowledge.

Also implied by the results of the analysis is the value of early placement of sealants. The data for caries rates for the molars suggest that the best time to seal the teeth that could be "successes" is as soon as the tooth has erupted enough to allow effective isolation. The effect of delay is to reduce the number of "successes" that are available, and thus to decrease the anticipated savings ratios. Procedures to encourage early sealing, such as limiting the benefit to the first two years after expected eruption, should be part of a sealant benefit program. A set of standards, compatible with the findings of this analysis, have already been suggested (15).

\section{Conclusions}

1. Sealants as a covered benefit for molars may not cause premiums to increase if fees and copayment levels are properly balanced. If caries in children continues to decline, higher premiums may be required for groups requesting a sealant benefit.

2. Sealants as a covered benefit for bicuspids are likely to require higher overall premiums.

3. The levels of dental caries in the population should be monitored closely, because a further substantial decline in caries prevalence could diminish the economic argument for sealants.

\section{References}

1. Mertz-Fairhurst EJ. Current status of sealant retention and caries prevention. J Dent. Ed 1984 Feb;48(Suppl):18-26.

2. Council on Dental Materials, Instruments, and Equipment. Proceedings of a conference on pit and fissure sealants: why their limited usage? Chicago: American Dental Association, 1981

3. Glasrud PH. Insuring private dental care: are sealants included? Am J Pub Health 1985 Mar;75:285-6.

4. National Center for Health Statistics. Plan and operation of the Health and Nutrition Examination Survey, United States, 197173, by H. Miller. Vital and Health Statistics. Series 1 Nos. 10a and 10b. DHEW Pub No (HSM) 73-1310. Health Services and Mental Health Administration, Washington DC: Government Printing Office, Feb 1973.

5. National Center for Health Statistics. Plan and operation of the Hispanic Health and Nutrition Examination Survey, 1982-84. Vital and Health Statistics. Series 1 . In preparation. Draft available from the author.

6. Eklund SA, Ismail Al. Time of development of occlusal and proximal lesions: Implications for fissure sealants. J Public Health Dent 1986 Spring;46:114-121.

7. Ryge G, Baskin $P$. The future of pit and fissure sealants. J Am Soc Prev Dent 1973 Jan-Feb;54-57.

8. National Center for Health Statistics: Special dental data tape from the Health and Nutrition Examination Survey, United States, 1971-74

9. Ismail, AI, and Burt, BA. Prevalence of dental caries and periodontal disease in Southwestern Hispanic children aged five to 17 years. Results from Southwestern HHANES, 1982-84. Submitted for publication, 1985

10. US Public Health Service, National Institute of Dental Research, National Caries Program. The prevalence of dental caries in United States children, 1979-1980. Washington DC: Government Printing Office, 1981; NIH Publ no 82-2245.

11. Bureau of Economic and Behavioral Research. Dental fees charges by general practitioners and selected specialists in the United States, 1982. J Am Dent Assoc 1984 Nov;108:83-7.

12. Maryniuk GA. In search of treatment longevity-a 30 year perspective. J Am Dent Assoc 1984 Nov;109:739-44

13. Burt BA. Fissure sealants: clinical and economic factors. J Dent Educ 1984 Feb;48(Suppl):96-102.

14. Stamm JW, Bohannan MH, Graves RC, Disney JA. The efficiency of caries prevention with weekly fluoride mouthrinses. J Dent Educ 1984 48:617-24.

15. Burt BA. Cost-effectiveness of sealants in private practice and standards for use in prepaid care. J Am Dent Assoc 1985 Jan;110(1):103-7. 